\title{
$\checkmark$ Research Square \\ The Barriers in the implementation of Antimicrobial Stewardship Programs: A systematic Review
}

\author{
Nehad Ahmed ( $\nabla$ n.ahmed@psau.edu.sa ) \\ Prince Sattam bin Abdulaziz University College of Pharmacy \\ Iffanullah Khan \\ Universiti Sains Malaysia - Kampus Kesihatan \\ Mohamed Azmi Hassali \\ Universiti Sains Malaysia \\ Abdul Haseeb \\ Umm Al-Qura University \\ Amer Hayat Khan \\ Universiti Sains Malaysia
}

Research article

Keywords: Antimicrobial, stewardship, Barriers

Posted Date: September 12th, 2019

DOI: https://doi.org/10.21203/rs.2.14377/v1

License: (c) (1) This work is licensed under a Creative Commons Attribution 4.0 International License.

Read Full License 


\section{Abstract}

Background The resistance to Antimicrobial is considered as one of the most important risks that affect public health and healthcare. Antimicrobial stewardship programs comprise a series of interventions that help to stop the inappropriate use of antimicrobials.

Methods The published literature on the barriers of implementing stewardship programs is limited. We searched for the relevant articles from 2007 till the end of 2017 using the keyword "Antimicrobial Stewardship Programs" in PubMed database.

Results There are many barriers in the implementation of antimicrobial stewardship programs. To improve the rational use of antibiotics it is important to overcome these barriers.

Conclusion According to the included studies into current review or manuscript, there are 9 major barriers for ASPs. Lack of training and knowledge, inter-professional and inter-specialty conflict, and lack of resources are the most common barriers.

\section{Background}

The resistance to Antimicrobials is considered as one of the most important risks that affect the public healthcare. Approximately $20 \%-50 \%$ of antibiotic use is inappropriate (1). The practice of antimicrobial stewardship programs (ASPs) in hospitals results in improving the use of antibiotics as well as decreasing of the antibiotic resistance (2). In addition to that, the effective antibiotic stewardship program can help in decreasing the treatment cost due to inappropriate prescriptions. (3).

Antimicrobial stewardship programs apply a series of interventions for monitoring and guidance to their appropriate use at health care institutions. It leads to provide an evidence-based approach in using antimicrobials (4). These programs are designed to encourage the proper use of antimicrobials by optimizing the selection of the antimicrobial agent, required dose, suitable route, and the duration of the treatment (5).

The successful implementation of Antimicrobial stewardship programs needs careful attention to the main barriers (6). There are many hurdles for antimicrobial stewardship programs which lead to the inappropriate prescription. It is important to know and overcome these shortcomings to improve the use of antibiotics. Therefore, the aim of this review is to review the common barriers for the implementation of antimicrobial stewardship programs.

\section{Methods}

The published literature on the barriers of implementing ASPs is limited. We searched for the relevant articles from 2007 till the end of 2017 using the keyword "Antimicrobial Stewardship Programs" in 
PubMed database. We identified 672 studies and limit the search to the last 10 years' full-text articles, which further decreased the total number of articles to 618 .

After that we limit the search to include human study only so we exclude another 200 articles. Finally, we limit the search accordingly to our manuscript title, "Antimicrobial Stewardship Programs AND Barriers" using AND tool and included only 31 studies. The flow diagram for a literature search is shown in figure 1 and the summary of the published studies regarding ASPs barriers is shown in table 1 and figure 2 .

\section{Results And Discussion}

\section{The Barriers in the implementation of Antimicrobial Stewardship Programs}

\section{The erratic clinical workflow in specific settings such as workload, time constraints, and decision fatigue}

The erratic clinical workflow is found in specific settings especially in the emergency department because of the rapid pace in it, which can possibly lead to decrease patient and family participation in the care plan, communication between the health care providers with their patients, and limit the time available for a health care provider for reading and doing research activities $(4,7,8,9)$.

Another obstacle is the increasing workload. If the health care providers are getting tired, the quality of their decisions will be deteriorated (7).

\section{Patient pressure and satisfaction}

Physicians sometimes prescribe antibiotics for patients, despite disagreeing with the need for antibiotics, to increase patient's satisfaction and also prevent losing of patients to other physicians for antibiotic prescription $(7,10)$.

\section{Diagnostic Barriers}

Another barrier for ASPs implementation are diagnostic barriers. For example, if the causative organism of infection is either virus or bacteria and there is difficulty in diagnosis, it can lead to excessive use of antibiotics (11). In addition to that many long-term care facilities do not have onsite microbiology laboratories and it will also delay the results of organism identification and susceptibility tests (10). Lack of microbiology services is also reported in another study as a barrier (12). 


\section{Social barriers, logistic and ethical concerns}

Social barriers such as perception of resistance, mistrust in antibiotic guidelines, and the skepticism of antimicrobial stewardship importance also affect the application of ASPs. $(6,11,15)$ Other studies reported that sociocultural beliefs, logistic barriers, and ethical concerns put impact in the implementation of $\operatorname{ASPs}(8,13)$.

Many pharmacists perceived a logistic barrier to provide clinical support, in particular, the limited communication with other staff, inadequate access to patient' clinical information, and the logistical issues of off-site General Physicians and pharmacists (14). Lack of willingness to change and perceived loss of prescribing autonomy are other examples reported in other studies $(12,16)$.

\section{Hospital hierarchies}

One of the significant prescribing barriers is hierarchical structures $(6,11,17)$. The study reported that the hospital hierarchies and consultation etiquette result in conflict between several physicians. For example, in respiratory tract infections, there are conflicts between respiratory specialists and infectious disease specialists. Another example for hierarchical structures barrier is the approval processes which may involve senior physicians to take approval from more junior antimicrobial stewardship physician which is considered as insult (18).

\section{Lack of training and knowledge}

Lack of knowledge and training can result in inappropriate prescribing of antibiotics $(2,3,4,5,9,10,12$, $16,19,20,21,22,23,24)$. One study reported that the lack of knowledge and education for nurses working in respiratory clinics represents a major barrier for the application of ASPs (18). Another study stated that one of the most important problems in the implementation of ASPs is the lack of knowledge regarding the fundamental rules on which antibiotics prescription are based (25). The availability of many clinical guidelines result into lack of knowledge because of the reason that clinicians cannot update themselves with all of these guidelines (1).

\section{Communication between microbiology laboratories and clinical units}

The poor communication between clinical units and microbiology laboratories is considered one of the main barriers to ASPs implementation. The problems in reporting test results, limited provision of laboratory services and poorly integrated IT systems are examples for this barrier. $(26,27)$ 


\section{Inter-professional and inter-specialty Conflict}

It is one of the important barriers in APSs and includes conflict regarding the responsibilities for different health care professionals, opposition from prescribers, the communication with antagonizing colleagues, and heterogeneity in the prescribing practices, refuse to accept policies, guidelines about use of antimicrobials, and clinical misconceptions. In addition to that, the limited contact between the health care professionals and the relations between them may compromise on the success of $\operatorname{ASPs}(4,5,9,14$, $17,18,19,20,21,23,24,27,28,29,30)$.

\section{Lack of resources}

This barrier includes lack of information technology, audit feedback, guidelines, funding, and lack of human resource.

Lack of information technology included inaccurate or incomplete data access and data extraction. (19, $21,31)$. Another barrier is the lack of ASPs electronic surveillance programs and the lack in clinical decision support tools $(8,27)$. One study reported that lack of guidelines is also one of the important barrier $(5,9,10)$. Other studies reported that barriers in the implementation of ASPs include the large volume of audits that are required to assess antibiotic use, lack of resources, enforcement, and specialist support. $(12,16,27,32)$.

Lack of funding and human resource is another barrier reported by several studies $(10,12,15,19,21,22$, $23,24,27,30)$ in addition to that high level of transient staff $(12,16)$, the lack of dedicated pharmacy and medical staff $(15,16)$, and the staffing constraints $(15,27)$.

Other barriers include lack of leadership $(12,16,23)$, pharmacy resources, and microbiology resources $(16,22,27)$. Table 2 summarizes the barriers in the implementation of Antimicrobial Stewardship Programs.

Centers for Disease Control and Prevention mentioned many barriers that can lead to inappropriate antibiotics prescription which is categorized according to it into 4 groups:

1. Lack of knowledge of appropriate indications

2. Diagnostic uncertainty and fear of complications

3. Patient pressure and satisfaction

4. Habit, workload, time constraints, and decision fatigue

\section{Conclusions}

ASPs play a significant role to decrease bacterial resistance and to improve use of antibiotics.

Unfortunately, there are many barriers that affect the implementation of these programs. According to the 
included studies into current review or manuscript, there are 9 major barriers for ASPs. Lack of training and knowledge, inter-professional and inter-specialty conflict, and lack of resources are the most common barriers. In order to implement these programs appropriately, there is dire need to know and overcome these barriers. The current review will be of interest and update the knowledge in determining the main barriers in order to overcome these barriers and to assist in the appropriate implementation of ASPs.

\section{Abbreviations}

Antimicrobial stewardship programs (ASPs)

\section{Declarations}

- Ethics Approval and Consent to Participate

Not applicable

- Consent to publish

Not applicable

- Availability of data and materials

All data generated or analyzed during this study are included in this published article [and its supplementary information files]

- Competing interest

None of the authors have any competing interests

- Funding

Not applicable

- Authors' contributions

NAanalyzed and interpreted the data and was a contributor in writing the manuscript. Other authors complete writing and make a revision for the manuscript. All authors read and approved the final manuscript.

- Acknowledgements

Not applicable

\section{References}


1. Appropriate Antibiotic Use | Antibiotic Use | CDC [Internet]. Cdc.gov. 2018 [cited 21 December 2018]. Available from: https://www.cdc.gov/antibiotic-use/index.html

2. Stenehjem E, Hyun D, Septimus E, Yu K, Meyer M, Raj D Et al. Antibiotic Stewardship in Small Hospitals: Barriers and Potential Solutions. Clinical Infectious Diseases. 2017;65(4):691-696.

3. Ravi N, Laha A, Hmar L, Chatterjee S, Goswami J, Goel G, Dhar K, Ghosh T, Chatterjee S, Datta SS, Bhattacharya S. Exploring the prescribing behaviors and the mind of antibiotic prescribers is critical for a successful antibiotic stewardship programme: Results of a survey from Eastern India. Indian Journal of Medical Microbiology.2017;35:299-301

4. Pranitha, D. Tamma. Sara, E. Cosgrove. Antimicrobial Stewardship. Infectious Disease Clinics of North America. 2011; Volume 25, Issue 1, 245-260

5. Nagel, JL., Kaye, KS., LaPlante, KL., Pogue, JM. Antimicrobial stewardship for the infection control practitioner. Infectious Disease Clinics of North America. 2016; 30:771-784.

6. Mack M, Rohde J, Jacobsen D, Barron J, Ko C, Goonewardene M Et al. Engaging hospitalists in antimicrobial stewardship: Lessons from a multihospital collaborative. Journal of Hospital Medicine. 2016;11(8):576-580.

7. Chung P, Scandlyn J, Dayan P, Mistry R. Working at the intersection of context, culture, and technology: Provider perspectives on antimicrobial stewardship in the emergency department using electronic health record clinical decision support. American Journal of Infection Control. 2017;45(11):1198-1202.

8. Acquisto N, Baker S. Antimicrobial Stewardship in the Emergency Department. Journal of Pharmacy Practice. 2011;24(2):196-202.

9. Chaves N, Cheng A, Runnegar N, Kirschner J, Lee T, Buising K. Analysis of knowledge and attitude surveys to identify barriers and enablers of appropriate antimicrobial prescribing in three Australian tertiary hospitals. Internal Medicine Journal. 2014;44(6):568-574.

10. Morrill H, Caffrey A, Jump R, Dosa D, LaPlante K. Antimicrobial Stewardship in Long-Term Care Facilities: A Call to Action. Journal of the American Medical Directors Association. 2016;17(2): 183.e1-183.e16.

11. Broom J, Broom A, Kirby E, Gibson A, Post J. Clinical and social barriers to antimicrobial stewardship in pulmonary medicine: A qualitative study. American Journal of Infection Control. 2017;45(8):911916.

12. James R, Luu S, Avent M, Marshall C, Thursky K, Buising K. A mixed methods study of the barriers and enablers in implementing antimicrobial stewardship programmes in Australian regional and rural hospitals. Journal of Antimicrobial Chemotherapy. 2015;70(9):2665-2670.

13. Paño-Pardo J, Schüffelmann-Gutiérrez C, Escosa-García L, Laplaza-González M, Moreno-Ramos F, Gómez-Gil R et al. Opportunities to improve antimicrobial use in paediatric intensive care units: a nationwide survey in Spain. Clinical Microbiology and Infection. 2016;22(2):171-177.

14. Lim C, Kwong M, Stuart R, Buising K, Friedman N, Bennett N et al. Antimicrobial stewardship in residential aged care facilities: need and readiness assessment. BMC Infectious Diseases. 
2014;14(1).

15. Doron S, Nadkarni L, Lyn Price L, Kenneth Lawrence P, Davidson L, Evans J et al. A Nationwide Survey of Antimicrobial Stewardship Practices. Clinical Therapeutics. 2013;35(6):758-765.e20.

16. Bryant P. Antimicrobial stewardship resources and activities for children in tertiary hospitals in Australasia: a comprehensive survey. The Medical Journal of Australia. 2015;202(3):134-138.

17. Broom A, Broom J, Kirby E, Plage S, Adams J. What role do pharmacists play in mediating antibiotic use in hospitals? A qualitative study. BMJ Open. 2015;5(11):e008326.

18. Broom J, Broom A, Kirby E, Gibson A, Post J. How do hospital respiratory clinicians perceive antimicrobial stewardship (AMS)? A qualitative study highlighting barriers to AMS in respiratory medicine. Journal of Hospital Infection. 2017;96(4):316-322.

19. Bedi N, Gupta P. Antimicrobial stewardship in pediatrics: An Indian perspective. Indian Pediatrics. 2016;53(4):293-298.

20. Nathwani D, Lawson W, Dryden M, Stephens J, Corman S, Solem C Et al. Implementing criteria-based early switch/early discharge programmes: a European perspective. Clinical Microbiology and Infection. 2015;21: S47-S55.

21. Howard P, Pulcini C, Levy Hara G, West R, Gould I, Harbarth S et al. An international cross-sectional survey of antimicrobial stewardship programmes in hospitals. Journal of Antimicrobial Chemotherapy. 2014; 70(4):1245-55.

22. Avent M, Hall L, Davis L, Allen M, Roberts J, Unwin S et al. Antimicrobial stewardship activities: a survey of Queensland hospitals. Australian Health Review. 2014;38(5):557.

23. Owens R, Shorr A, Deschambeault A. Antimicrobial stewardship: Shepherding precious resources. American Journal of Health-System Pharmacy. 2009;66(12_Supplement_4):S15-S22.

24. Enani M. The antimicrobial stewardship program in the Gulf Cooperation Council (GCC) states insights from a regional survey. Journal of Infection Prevention. 2016;17(1):16-20.

25. Principi N, Esposito S. Antimicrobial stewardship in pediatrics. BMC Infectious Diseases. 2016;16(1).

26. Skodvin B, Aase K, Brekken A, Charani E, Lindemann P, Smith I. Addressing the key communication barriers between microbiology laboratories and clinical units: a qualitative study. Journal of Antimicrobial Chemotherapy. 2017;72(9):2666-2672.

27. Pakyz A, Moczygemba L, VanderWielen L, Edmond M, Stevens M, Kuzel A. Facilitators and barriers to implementing antimicrobial stewardship strategies: Results from a qualitative study. American Journal of Infection Control. 2014;42(10): S257-S263.

28. Broom J, Broom A, Plage S, Adams K, Post J. Barriers to uptake of antimicrobial advice in a UK hospital: a qualitative study. Journal of Hospital Infection. 2016;93(4):418-422.

29. Broom A, Plage S, Broom J, Kirby E, Adams J. A qualitative study of hospital pharmacists and antibiotic governance: negotiating interprofessional responsibilities, expertise, and resource constraints. BMC Health Services Research. 2015;16(1). 
30. Hamilton, KW, Fishman, NO. Antimicrobial stewardship interventions: thinking inside and outside the box. Infectious Disease Clinics of North America. 2014; 28:301-313.

31. Micallef C, Chaudhry N, Holmes A, Hopkins S, Benn J, Franklin B. Secondary use of data from hospital electronic prescribing and pharmacy systems to support the quality and safety of antimicrobial use: a systematic review. Journal of Antimicrobial Chemotherapy. 2017;72(7):18801885.

32. Broom A, Gibson A, Broom J, Kirby E, Yarwood T, Post J. Optimizing antibiotic usage in hospitals: a qualitative study of the perspectives of hospital managers. Journal of Hospital Infection. 2016;94(3):230-235.

\section{Tables}

Due to technical limitations, Table(s) 1 and 2 are only available as a download in the supplemental files section.

\section{Figures}



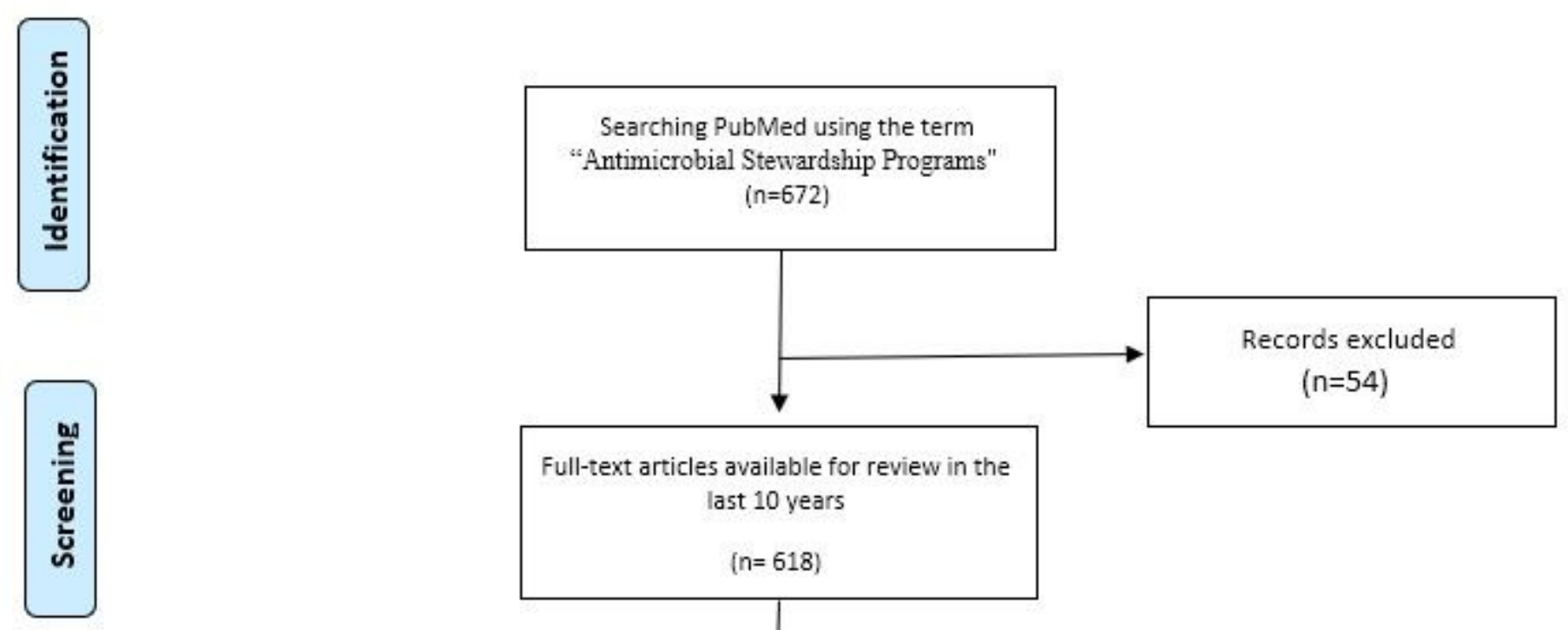

Full-text articles available for review in the last 10 years

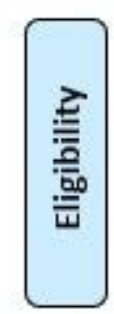

$(n=618)$

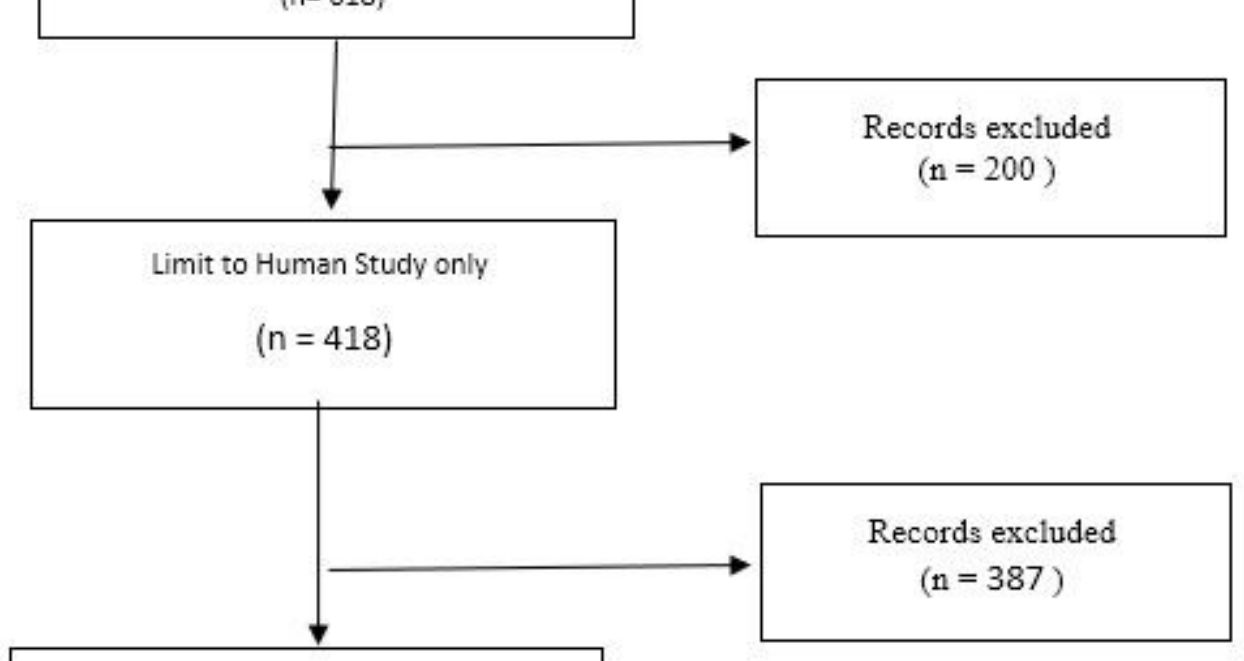

엄

Using "AND" tool ("Antimicrobial Stewardship Programs AND Barriers")

$$
\text { (n }=31 \text { ) }
$$

\section{Figure 1}

PRISMA chart of the search method. This figure shows the number of articles included in the review after limit the searching results 


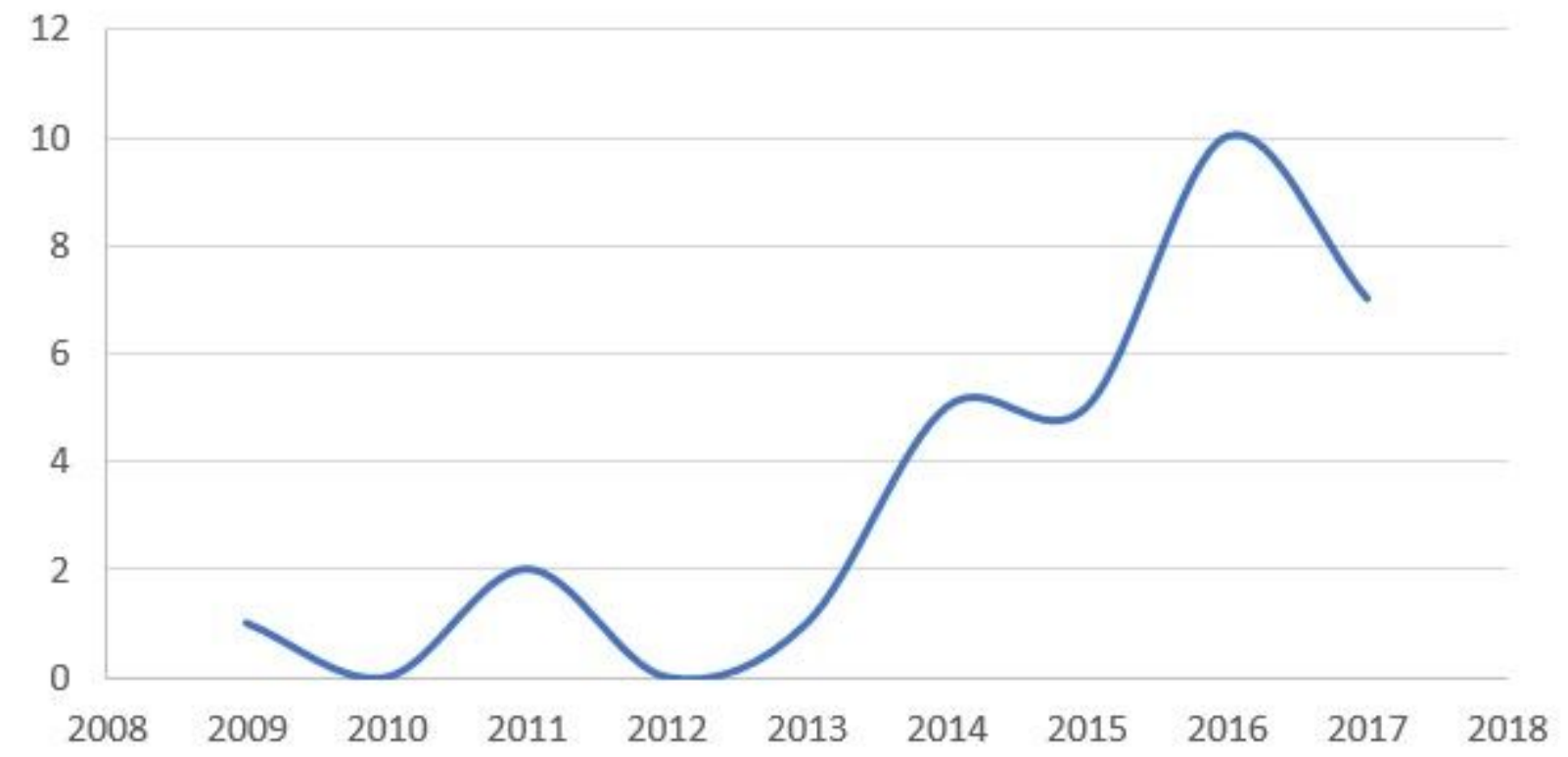

Figure 2

PubMed citations on the barriers of antimicrobial stewardship programs from the 2009 till 2017

\section{Supplementary Files}

This is a list of supplementary files associated with this preprint. Click to download.

- Table2.jpg

- PRISMA2009checklist.doc

- Table1.jpg 